\title{
Directional Elastic Wave Propagation in High-Aspect-Ratio Photoresist Gratings: Liquid Infiltration and Aging
}

\author{
E. Alonso-Redondo, ${ }^{1}$ A. Gueddida, ${ }^{2,3} \mathrm{~J} . \mathrm{Li},{ }^{4}$ B. Graczykowski, ${ }^{5}$ C. M. Sotomayor Torres, ${ }^{5,6}$ \\ Y.Pennec, ${ }^{2}$ S. Yang, ${ }^{4}$ B. Djafari-Rouhani, ${ }^{2}$ and G. Fytas ${ }^{1,7, *}$ \\ ${ }^{1}$ Max Planck Institute for Polymer Research, Ackermannweg 10, 55128 Mainz, Germany \\ ${ }^{2}$ Institut d'Electronique, de Microélectronique et de Nanotechnologie (IEMN), UMR-CNRS 8520, \\ UFR de Physique, Université de Lille 1, 59655 Villeneuve d'Ascq, France \\ ${ }^{3}$ LPMR, Département de Physique, Faculté des Sciences, Université Mohamed I, 60000 Oujda, \\ Morocco \\ ${ }^{4}$ Department of Materials Science and Engineering, University of Pennsylvania, 3231 Walnut Street, \\ Philadelphia, PA 19104, United States \\ ${ }^{5}$ Catalan Institute of Nanoscience and Nanotechnology_(ICN2), CSIC and the Barcelona Institute of \\ Science and Technology, Campus UAB, 08193 Bellaterra, Spain \\ ${ }^{6}$ ICREA, Pg. Lluís Companys 23,, 08010 Barcelona, Spain \\ ${ }^{7}$ Department of Materials Science, University of Crete and IESL/FORTH, 71110 Heraklion, Greece \\ *Corresponding author's email: fytas@mpip-mainz.mpg.de
}

\begin{abstract}
Determination of the mechanical properties of nanostructured soft materials and their composites in a quantitative manner is of great importance to improve the fidelity in their fabrication and to enable subsequent reliable utility. Here, we report on the characterization of the elastic and photoelastic parameters of a periodic array of nanowalls
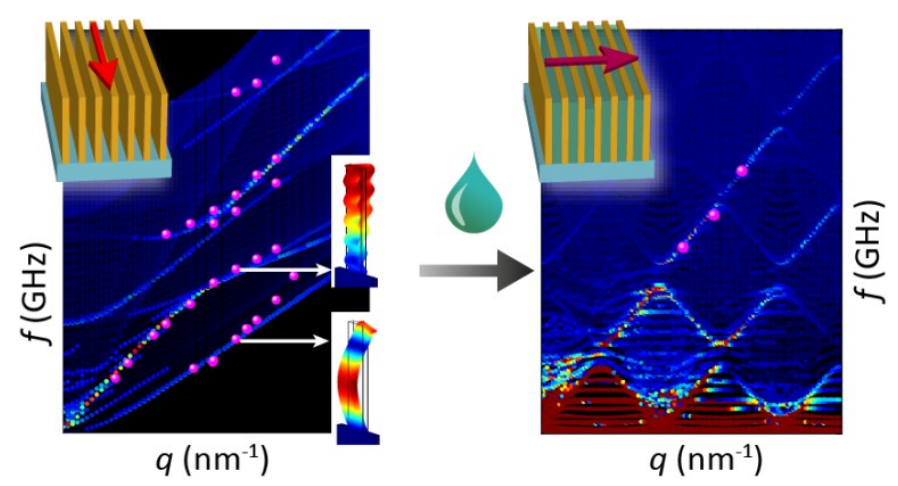
(grating) by the non-invasive Brillouin light scattering technique and finite element calculations. The resolved elastic vibrational modes in high and low aspect ratio nanowalls reveal quantitative and qualitative differences related to the two-beam interference lithography fabrication and subsequent aging in ambient conditions. The phononic properties, namely the dispersion relations, can be drastically altered by changing the surrounding material of the nanowalls. Here we demonstrated that liquid infiltration turns the phononic function from a single-direction phonon-guiding to an anisotropic propagation along the two orthogonal directions. The susceptibility of the phononic behavior to the infiltrating liquid can be of unusual benefits, such as sensing and alteration of the materials under confinement.
\end{abstract}


Thin and patterned polymer films with nanoscopic thicknesses are materials with growing interest, as the demand of miniaturization by the industry keeps on. Thin polymer structures are used in a variety of emerging applications including flexible electronics, ${ }^{1,2}$ superhydrophobic coatings, ${ }^{3,4}$ phononic crystals ${ }^{5}$ and plasma etching masks. ${ }^{6}$ The polymer nanostructures pose advantages in respect to their hard (e.g. silicon, metal) counterparts as they can be transparent, biocompatible and low cost. ${ }^{7}$ One dimensional (1D) gratings consisting on periodic arrays of nanowalls with high aspect ratio ( $A R=$ height / width) are desired in many applications. ${ }^{8}$ For example, as plasma etching mask, high $A R$ polymer nanowalls increase the etching resistance $;{ }^{9}$ therefore the diffraction efficiency of the resulting grating is improved ${ }^{6}$ However, the increasing compliance also limit the maximum $A R$ of nanowalls achievable, due to collapsing of the structures during fabrication or post-applications. ${ }^{10}$

The direct fabrication of high $A R(>2)$ polymer gratings has been elusive by photo- and nanoimprint lithography using conventional polymers. ${ }^{11}$ Organosilicate photoresists have enhanced the mechanical properties of the gratings, for the fabrication of stable ultrahigh $A R$ (up to 10) by interference lithography. ${ }^{12}$ The mechanical properties of the resulting nanowalls differ from the bulk depending on the degree of polymerization, post-exposure baking and rinsing processes. These nanowalls are amenable to buckling by boundary forces resulting from dissimilar expansion of the sample to the substrate. ${ }^{13}$ Also, capillary forces during drying of patterned lithographic films from developer lead to pattern collapse if the $A R$ is high or the material is mechanically weak. ${ }^{14}$ The stability and mechanical properties of nanostructures is of paramount importance to guarantee their optimal performance. Therefore it is requisite to develop methods to the precisely determination of the mechanical behavior of nanoscopic polymer structures.

The state-of-the-art methods for nanomechanic measurements are based on deformation, ${ }^{15,16}$ or on acoustic wave propagation. ${ }^{17,18}$ Both methods pose limitations: the former is destructive, while the latter requires a complex sample preparation. Both assume linear stress-strain relationship, ${ }^{19}$ and cannot resolve the direction-dependent mechanical behaviors. ${ }^{20}$ Yet, in the case of deformation tests, the estimation of the Young's modulus is based on the assumed value of the Poisson's ratio. The frequently employed atomic force microscopy (AFM) indentation technique suffers from drawbacks originating from small contact forces in the probe-sample interaction, and the influence of the rigid substrate. ${ }^{21}$ In this context, Brillouin light scattering (BLS) emerges as a robust technique that provides an alternative for the non-invasively determination of the direction-dependent mechanical properties at nanoscale, without complicated sample preparation. In BLS, the analysis of the spectral distribution of the scattered light by thermal phonons provides information about the mechanical properties of polymer thin films in their elastic response 
regime $(\mathrm{GHz}){ }^{22}$ In the case of supported films, the influence of the supporting substrate is reliably predicted by the theory. ${ }^{23}$

Here, we estimate the direction-dependent mechanical properties of high $A R$ nanoscopic gratings using BLS. The recorded spectra (frequency and intensity) are function of the geometric, elastic and photoelastic parameters. Through finite element method (FEM) modeling we access to the complete characterization of the 1D grating consisting of an array of nanowalls. Because the susceptibility of nanowall collapsing is dependent on the aspect ratio, ${ }^{13,24}$ we evaluate the size-dependent fidelity on high $A R$ and low $A R$ nanowalls. The direction-dependence is assessed in both, pristine and buckled-defect gratings. Fundamental understanding of the mechanical properties of nanoscopic nanowalls will provide insights how to improve the material performance, and realize the tuning of these sensitive materials by infiltrating liquid in the interstices of the grating. The presence of periodicity in the sub micrometer range invokes elastic wave (phonon) interactions in hypersonic frequencies that inevitably leads to deviations from the linear acoustic dispersion -frequency $f$ versus wave vector $\mathbf{q}$ - that is a characteristic feature of homogeneous bulk materials. Record of the rich dispersion $f(\mathbf{q})$ is necessary to unveil phononic behaviors that essentially determine the flow of the elastic energy in nanostructured materials. Therefore, in addition to the nanomechanic characterization, the phononic character of the grating is assessed in both pristine and liquid filled structures.

\section{- RESULTS AND DISCUSSION}

The 1D gratings consisting of a periodic array of soft nanowalls were fabricated by two-beam interference lithography (Methods) with two distinct $A R=11$ (nanowalls, Figure 1) and $A R=2$ (nanolines). ${ }^{25}$ In brief, the photoresist film was prepared from epoxy polyhedral oligomeric silsesquoxane (epoxy-POSS), a transparent organosilicate consisting of POSS molecules (smallest silica particles) with covalently bonded epoxy groups suitable for photopolymerization. The spincoated film was exposed to a 532nm-laser, and baked at $50{ }^{\circ} \mathrm{C}$ to crosslink the exposed regions. Then, the grating was developed in propylene glycol monomethyl ether acetate. The geometric parameters height $h$, width $w$ and period $a$ were determined by scanning electron microscopy (SEM) and are listed in Table 1. Nanowalls and nanolines are prepared directly on glass substrates, therefore the nanowalls are not in contact through any bottom layer. ${ }^{26,27}$

Table 1. Characteristic dimensions of the photoresist gratings. ${ }^{\mathrm{a}}$

\begin{tabular}{lccccc}
\hline Sample & $h(\mathrm{~nm})$ & $w(\mathrm{~nm})$ & $A R$ & $a(\mathrm{~nm})$ & $w / a$ \\
\hline Nanolines & 600 & 320 & 2 & 650 & 0.5 \\
Nanowalls & 2700 & 250 & 11 & 630 & 0.4
\end{tabular}

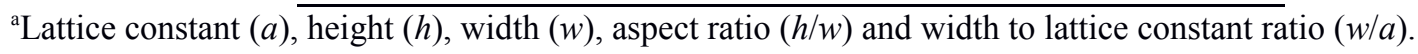



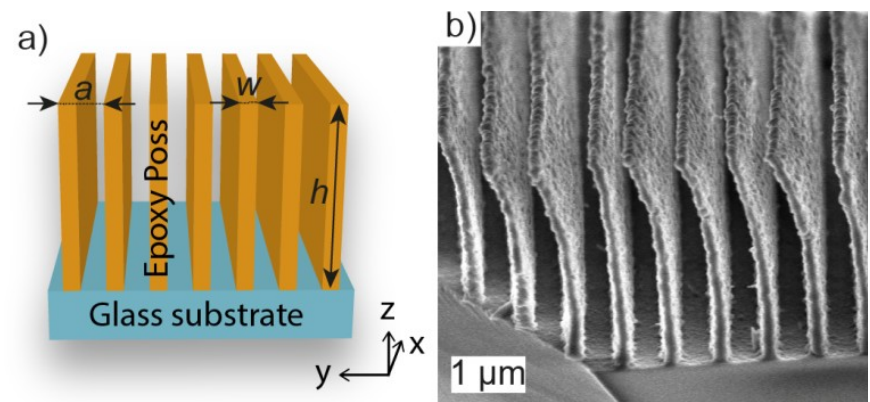

Figure 1: Structure of the samples. (a) Schematic illustration of a 1D-grating with lattice constant $a$, width $w$, and height $h$ along with the principal axes. (b) Scanning electron microscope (SEM) images of nanowalls $(A R=11)$; the bending is due to the electron beam during imaging by SEM.

\section{High $A R$ nanowalls}

Direct measurements of elastic properties in soft gratings are performed with the non-contact and nondestructive BLS technique. BLS spectroscopy is based on the photoelastic interaction of thermal hypersonic $(\mathrm{GHz})$ elastic excitations with the probing laser beam. Thermal phonons interact with the photons to give rise to a momentum transfer manifested as frequency shifts in the recorded spectrum of scattered light. The momentum transfer between the incident photon and an elastic single mode (phonon) is expressed by $\hbar \mathbf{q}=\hbar \mathbf{k}_{\mathrm{s}} \pm \hbar \mathbf{k}_{\mathrm{i}}$, where $\hbar \mathbf{k}_{\mathrm{i}}$ and $\hbar \mathbf{k}_{\mathrm{s}}$ are the momenta of the incident and scattered photons, respectively. The spectral Brillouin shift, $f$, depends on the direction and magnitude of the scattering wave vector $\mathbf{q}$ as described in the dispersion relation $f(\mathbf{q})$. For transmission geometry (Figure 2a), the magnitude of the wave vector is $q=4 \pi / \lambda_{\mathrm{T}} \cdot \sin (\theta / 2)\left(\lambda_{\mathrm{T}}=532 \mathrm{~nm}, \theta\right.$ is the scattering angle) and for backscattering geometry (inset Figure 2e) is $q=4 \pi / \lambda_{\mathrm{BS}} \cdot \sin \alpha\left(\lambda_{\mathrm{BS}}=514.5 \mathrm{~nm}\right)$, where $\alpha$ is the angle of the beam to the normal of the substrate. Responsible for BLS in transmission and backscattering geometries is the photoelastic and surface ripple mechanisms, respectively. The ripple mechanism is enhanced in this transparent material by a thin $(\sim 15 \mathrm{~nm})$ aluminum coat, which results in a weak blue shift $(<3 \%)$ of the phonon frequencies due to the structure stiffening, as confirmed by FEM calculations. ${ }^{28}$ In transmission geometry, the incident light is polarized vertically (V) to the scattering plane $x z$ (Figure 2a). The polarized spectra were recorded with a vertical (V) polarization, while the depolarized spectra were recorded with horizontal $(\mathrm{H})$ polarization (in the $x z$ plane). In the back-scattering experiment, the incident light is horizontally polarized, and the collected light is vertically polarized (HV). Polarized and depolarized light carry distinct information about longitudinal or transverse displacement modes, respectively. The dispersion $f(\mathbf{q})$ was recorded for phonon propagation along the nanowalls ( $x$-axis, Figure 2a) because along the periodicity direction ( $y$-axis) the strong diffracted beams obscures the BLS signal in the transmission geometry. The periodicity effect ( $p_{\mathrm{L}}$ and $p_{T}$ branches) is discussed in Supplementary Information. 

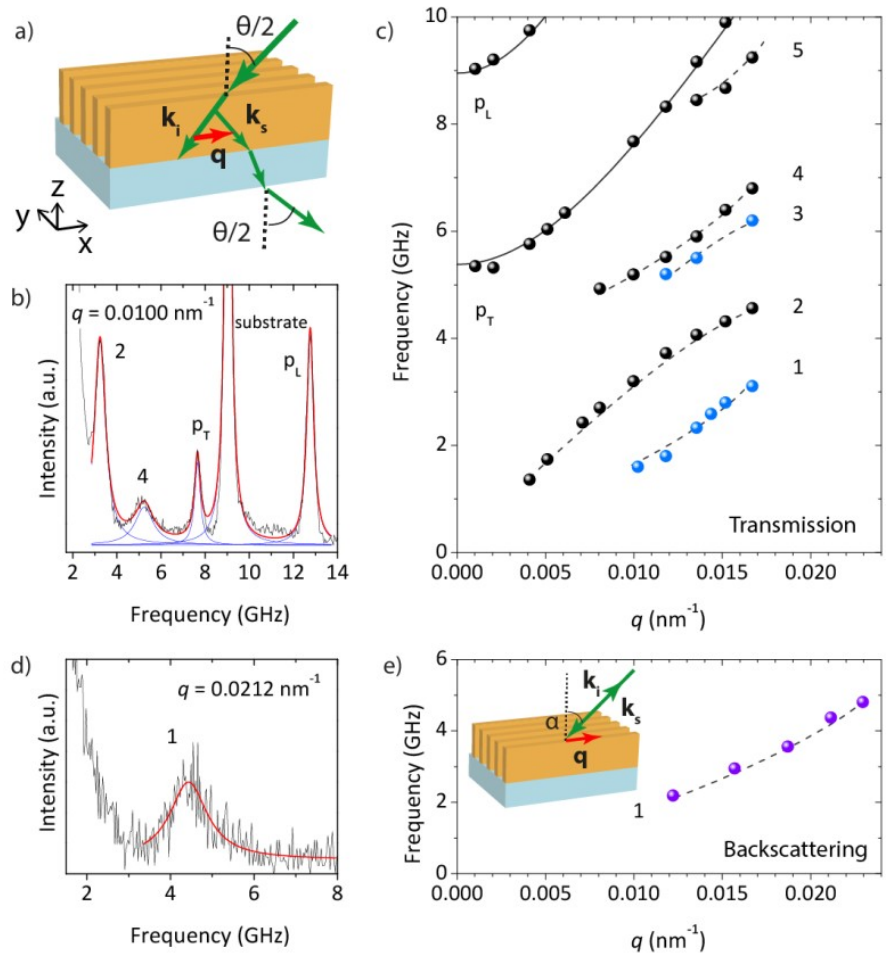

Figure 2: Brillouin light scattering of high aspect ratio nanowalls. (a) Schematic of the BLS scattering geometry (transmission). $\mathbf{k}_{\mathrm{i}}, \mathbf{k}_{\mathrm{s}}$ are the incident and scattered wave vectors respectively, oriented an angle $\theta$ respect to each other. $\mathbf{q}$ is the scattering wave vector along the nanowalls. (b) Experimental polarized (VV) BLS spectrum represented by a sum of Lorentzian shapes (blue and red solid lines). (c) Dispersion relation $f(\mathbf{q})$, acquired in VV (black circles) and $\mathrm{VH}$ (blue circles) polarizations. Solid lines denote the representation of $p_{\mathrm{L}}$ and $p_{\mathrm{t}}$ modes by Supplementary Eq. 1, while the dashed lines are guides for the eye. (d) Experimental backscattering spectrum represented by a Lorentzian shape (red line). (e) Dispersion relation acquired in backscattering geometry (inset) and HV polarization; dashed line is a guide for the eye.

The dispersion plot (Figure 2c) consist of five branches (1-5) related to the different elastic excitations within the photoresist gratings, along the nanowalls. The recorded spectra in transmission geometry consist of many peaks (representative spectrum in Figure 2b), and in backscattering geometry a single phonon was observed (Figure $2 \mathrm{~d}$ ). The dispersion relation $f(\mathbf{q})$ in Figure $2 \mathrm{c}$ consists of a collection of branches acquired in VV polarization (black) and VH polarization (blue). Branch 1 in Figure 2e was acquired with HV polarization in backscattering geometry. We note that a single sample was used to record the dispersion in each case; the reproducibility (within 1\%) is proven using an additional sample. The nature of the observed modes is disclosed by the theoretical representation of the full BLS spectrum.

We utilize the FEM analysis (Methods) to calculate the dispersion curves of elastic waves propagating along the nanowalls. Many modes are anticipated due to the finite size of the nanowalls and their attachment to a quasi-rigid substrate; however, only a discrete number of modes were observed in 
the experiment. To filter the modes that contribute strongly to the BLS intensity, we use the band sorting method (Supplementary Information and Figure S1) and the photoelastic coupling between the probing light and the sorted elastic modes. ${ }^{29}$ The dielectric tensor $\varepsilon_{\mathrm{jk}}$ at any given point is modulated by the elastic wave due to the strain tensor $S_{\mathrm{lm}}$ : $\delta \varepsilon_{\mathrm{jk}}=P_{\mathrm{jklm}} S_{\mathrm{lm}}$, where $P_{\mathrm{jkkm}}$ is the photoelastic (Pockels) tensor. In isotropic homogeneous media, only two photoelastic constants describe the materials, in Voigt notation $P_{11} \quad\left(=P_{1111}\right)$ and $P_{12} \quad\left(=P_{1122}\right){ }^{30} \quad$ The $\quad$ BLS intensity is given by $I=\left|E^{s}\right|^{2} \propto\left|\frac{1}{2 \pi f} \int_{V} e^{i \vec{a} \vec{r}} \sum_{k l m} P_{j k l m} S_{l m} E^{0} d V\right|^{2}$, where $E^{0}, E^{\mathrm{S}}$ are the incident and scattered electric fields, respectively, $V$ is the scattering volume and $i$ is the imaginary number. In our particular case where the propagation is along $x(\vec{r}=(1,0,0))$, the BLS intensities in $\mathrm{VV}$ and $\mathrm{VH}$ polarizations are $\mathrm{e}^{31}$

$$
\begin{gathered}
I_{V V} \propto\left|\frac{1}{2 \pi f} \int_{V} e^{i q x}\left(P_{12} \partial_{x} u_{x}+P_{11} \partial_{y} u_{y}+P_{12} \partial_{z} u_{z}\right) E^{0} d V\right|^{2} \\
I_{V H} \propto\left|\frac{1}{2 \pi f} \int_{V} e^{i q x} \frac{P_{11}-P_{12}}{2}\left[\cos \frac{\theta}{2}\left(\partial_{x} u_{y}+\partial_{y} u_{x}\right)+\sin \frac{\theta}{2}\left(\partial_{y} u_{z}+\partial_{z} u_{y}\right)\right] E^{0} d V\right|^{2}
\end{gathered}
$$

where $u_{i}$ is the displacement and $\partial_{\mathrm{j}}$ denotes a partial derivative along the $j$-th direction. The selection rules in Eqs. 2 and 3 allow only few modes to contribute to the BLS intensity in each polarization (Figure 3a and $3 \mathrm{~b})$. The theoretical spectra were convoluted with the instrumental width $(\sim 0.3 \mathrm{GHz})$. The excess of broadening in the experimental spectrum is due to additional damping because of phonon scattering and secondary relaxations in the glassy epoxy. The nanowall elastic and photoelastic parameters (in Table 2) are then obtained from the unique theoretical description of the full experiment (dispersion and peak intensities).

Figure $3 \mathrm{c}$ displays the theoretical BLS intensity $\left(I_{\mathrm{VV}}+I_{\mathrm{VH}}\right)$ dispersion relation. The bands with sufficiently large BLS intensity are colored, while the bands with zero intensity (dark blue) are set to a certain transparency for visualization purposes. A thorough inspection of the dispersion plot (Figure 3c) reveals that the experimental points can be satisfactorily described by the Lamb modes of an infinite plate of the same wall thickness, $w$ (Figure $\mathrm{S} 2$ ). Lamb modes are classified as $\mathrm{A}_{\mathrm{n}}$ and $\mathrm{S}_{\mathrm{n}}$, with antisymmetric and symmetric displacements of the plate free surfaces from their mid-plane, respectively. Based on the displacement field, $A_{n}$ modes are observed in VH (Figure 3b, Eq. 2), while $S_{n}$ modes are observed in VV (Figure 3a, Eq. 3) polarization. The fundamental modes $\mathrm{A}_{0}$ (flexural) and $\mathrm{S}_{0}$ (extensional) are the most important since they carry large fraction of the elastic energy and have no low cut-off frequency at $q=0$ (Figure 3c). The cut-off frequency of higher order modes is $f=m c / 2 w$, where $m$ is a positive integer, and $c$ is the epoxy's transverse velocity for even $A_{n}$ and odd $S_{n}$ modes, or the epoxy's longitudinal velocity for 
odd $A_{n}$ and even $S_{n}$ modes. The elastic parameters in Table 2 reveal bulk-polymer-like behavior of pristine high $A R$ nanowalls as both the Poisson ratio (0.32) and the longitudinal sound velocity $(2620 \mathrm{~m} / \mathrm{s})$ are values typical for glassy polymers. Access to exactly the same bulk material forming the gratings is not possible, since the fabrication involves a process of crosslinking by an interference pattern of variable intensity, and post-exposure baking and developing that affects the final properties. Hence, the nondestructive in situ characterization of the grating is crucial.
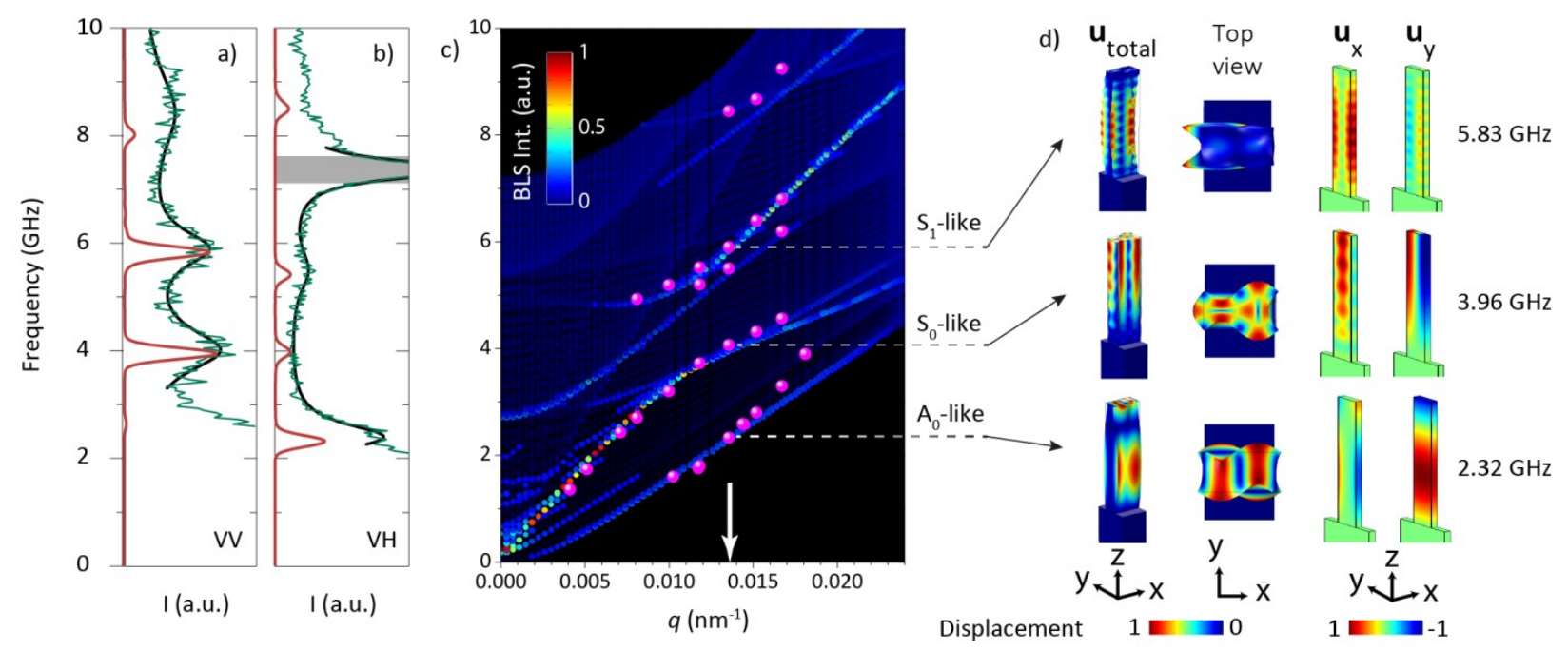

Figure 3: High aspect ratio nanowalls. (a-b) Experimental (green) and computed (red) spectrum at $q=0.0135 \mathrm{~nm}^{-}$ ${ }^{1}$ in VV and $\mathrm{VH}$ polarization. The experimental spectra have been represented by a sum of Lorentzian lines (black). The shaded region in (b) corresponds to the scattering peak of the glass substrate. (c) Computed BLS intensity (VV $+\mathrm{VH}$ polarizations) and experimental (pink circles) phononic band diagram. The arrow marks the wavenumber of the spectra in (a-b). (d) Normalized total ( $\left.\mathbf{u}_{\text {total }}\right)$ and selected displacement fields along $x\left(\mathbf{u}_{\mathrm{x}}\right)$ and $y\left(\mathbf{u}_{\mathrm{y}}\right)$ directions of a nanowall slice, at $q=0.0135 \mathrm{~nm}^{-1}$ and three frequencies as indicated in the plot. The total displacement has been exaggerated for visualization purposes.

Table 2. Values of the physical quantities used in the theoretical calculations. ${ }^{b}$

\begin{tabular}{lccccc}
\hline Parameter & Nanowalls & Nanolines & Glass substrate & Cargille & PDMS \\
\hline$\rho\left(\mathrm{kg} / \mathrm{m}^{3}\right)$ & 1190 & 1190 & 2200 & 1000 & 965 \\
$c_{L}(\mathrm{~m} / \mathrm{s})$ & 2620 & 2950 & 5660 & 1670 & 1000 \\
$c_{T}(\mathrm{~m} / \mathrm{s})$ & 1350 & 1450 & 3390 & 0 & 0 \\
$v$ & 0.32 & 0.34 & 0.22 & & \\
$E(G P a)$ & 5.29 & 6.20 & 617 & & \\
$P_{I I} / P_{12}$ & 0.100 & 0.667 & & & \\
\hline
\end{tabular}

${ }^{b}$ Density $(\rho)$, longitudinal $\left(c_{L}\right)$ and transverse $\left(c_{T}\right)$ sound velocities, Poisson's ratio (v), Young's modulus $(E)$ and photoelastic coefficients ratio $\left(P_{11} / P_{12}\right)$. 
The observed modes 1-5 in Figure $2 \mathrm{c}$ are identified as $\mathrm{A}_{0}, \mathrm{~S}_{0}, \mathrm{~A}_{1}, \mathrm{~S}_{1}$ and $\mathrm{S}_{2}$, respectively. The displacement fields of some of the modes that contribute to the BLS intensity are displayed in Figure $3 \mathrm{~d}$. These modes can be understood as guided modes along $x$ direction, and stationary in $y$ and $z$ directions. At low $q$ 's $\left(<2 \pi / w \sim 0.026 \mathrm{~nm}^{-1}\right)$, the $\mathrm{A}_{0}$-like mode (active in $\mathrm{VH}$ and $\mathrm{HV}$ polarizations) is dominated by the transverse $u_{\mathrm{y}}$, while the $\mathrm{S}_{0}$-like mode (active in $\mathrm{VV}$ ) is prevailed by the longitudinal $u_{\mathrm{x}}$ displacement. At high $q$ 's, the wavelength of the phonon is shorter than the wall thickness, and the $\mathrm{A}_{0}$ and $\mathrm{S}_{0}$ modes propagate on the vertical free surfaces of the walls with the speed of a Rayleigh wave of epoxy. Higher harmonics, corresponding to stationary waves along the height of the nanowall with their oscillations in the $z$-axis, contribute little to the BLS spectra (blue-transparent lines in Figure 3c). These modes become more important with the decrease in $A R$, and indeed their intensity is stronger in nanolines (Figure $4 \mathrm{~g}$ ).

\section{Aging effects}

Polymer gratings are amenable to age by factors such as humidity, stress and temperature. ${ }^{13,19,32,33}$ Since one of our goals is to tune the elastic properties of nanowalls by liquid infiltration, the study of possible shape deformation of the gratings under wet conditions is relevant. Nanowalls stored in the lab for a few months - under ambient temperature $\left(\approx 23{ }^{\circ} \mathrm{C}\right)$ and humidity- are buckled (Figure $\left.4 \mathrm{a}\right)$. It has been reported that nanowalls exposed to humidity absorb water and swell. ${ }^{34}$ Because the nanowalls are attached to a rigid substrate, residual stress along the lamina is created. When the critical stress $S^{*}$ is reached --depending on the nanowall aspect ratio --, straight nanowalls form wavy patterns. ${ }^{19}$

Since the elastic vibrations of the straight nanowalls can be described effectively by an infinite plate, the BLS intensities of the buckled structures are computed for the infinite plate instead of the finite nanowalls. The theoretical BLS intensities at several $q$ 's are displayed for three values of the amplitude $(\delta)$ and the period $(d)$ of the wavy patterns (inset to Figure $4 \mathrm{a}$ ) are displayed in Figure $4 \mathrm{~b}$ and Figure $4 \mathrm{c}$, respectively. The blue lines are for $d=8400 \mathrm{~nm}$ and $\delta=646 \mathrm{~nm}$ (observed values by SEM) and the green lines denote spectral shape for the straight plate $(\delta=0 \mathrm{~nm}$ or $\mathrm{d} \rightarrow \infty)$. In general, a variation of either $d$ or $\delta$ results in a diminution of the BLS intensities accompanied by a slight red-shift relative to straight plate case; to observe a significant red-shift, $\delta / d$ must be unrealistically small. As this zig-zag structure does not display a symmetry plane, more modes (than only $\mathrm{S}_{0}$-like and $\mathrm{S}_{1}$-like) can be involved in the scattering process. The results remain similar to the straight nanowalls as far as the ratio $\delta / d$ is small, while upon increasing $\delta / d$ more modes appear in the vibration spectrum. Therefore, a moderated buckling does not affect strongly the phonon propagation along the nanowalls. ${ }^{35}$ 

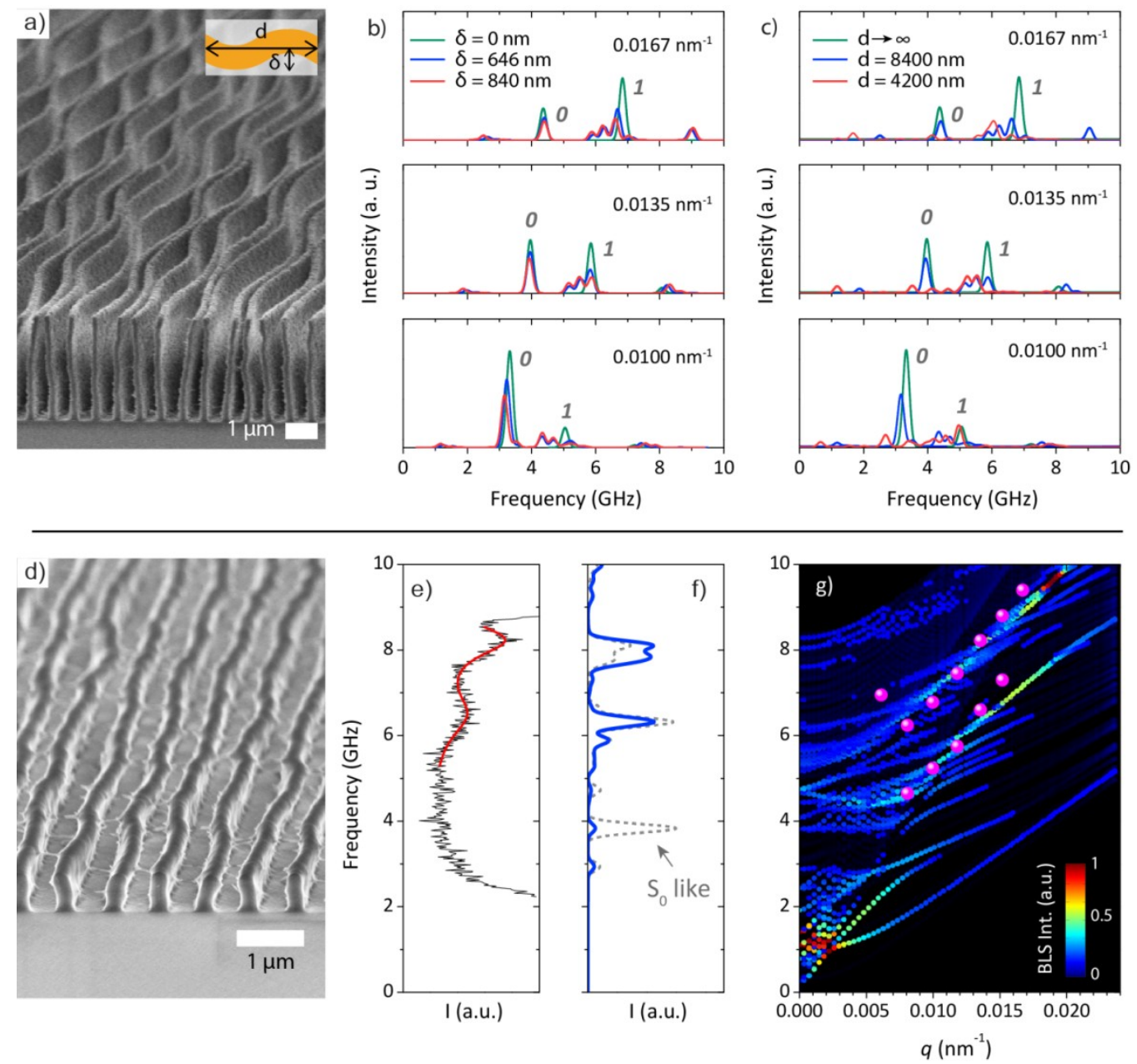

Figure 4: Aging effects. (a) Cross sectional SEM micrograph of nanowalls. (b-c) Computed VV BLS intensity of buckled infinite plate. The (b) amplitude $(\delta)$ and $\mathbf{c}$ ) period $(d)$ of the ondulation is varied around its mean value 646 $\mathrm{nm}$ and $8400 \mathrm{~nm}$ respectively (blue lines). The parameters were fixed to $d=8400 \mathrm{~nm}$ in (b) and $\delta=646 \mathrm{~nm}$ in (c). The intensity spectrum of a straight nanowall is shown (green lines); peaks identified as $\boldsymbol{O}$ and $\boldsymbol{1}$ correspond to the $\mathrm{S}_{0}$-like and $\mathrm{S}_{1}$-like modes, respectively. (d) Cross sectional SEM micrograph of nanolines. (e) Experimental (VV polarization) and (f) computed spectrum at $q=0.0135 \mathrm{~nm}^{-1}$ in nanolines. In (f) the theoretical spectra are calculated using $\mathrm{P}_{11} / \mathrm{P}_{12}=0.67$ (blue line) and (c) $\mathrm{P}_{11} / \mathrm{P}_{12}=0.10$ (grey dashed line). (g) Computed BLS intensity and experimental (pink circles) phononic band diagram of the nanolines in $\mathrm{VV}$ polarization.

The apparent touching of the buckled nanowalls (Figure 4a) could, in principle, alter the dispersion relation, and the phonon-guiding character. To evaluate precisely the influence of contact between walls, the dispersion relation for two walls touching in their tops was computed. No significant changes in the dispersion are found for one-point contact, or $1 / 4$ of the nanowall height $h$ (Figure S3). For a touching portion larger than $h / 2$, there are significant changes in the dispersion; the formed pseudo-plate behaves as a Lamb plate of $2 w$ thickness. Nevertheless, the latter scenario is highly unrealistic and is disregarded. Consequently, touching of the nanowalls is not expected to alter the phononic properties. However, the experimental dispersion relation of the aged gratings reveal changes at high $q$ 's (or short sizes) and the 
observed disparity deviation is ascribed to morphological issues due to fabrication (see Supplementary Information and Figures S4, S5, S6).

In order to test the role of nanowall height on the buckling effect, we measured the dispersion by BLS of short $A R(=2)$ nanolines. In contrast to the nanowalls, nanolines seem robust against buckling and there is no significant deformation (Figure 4d) when aged at the same conditions as the nanowalls. Assuming that $S^{*} \times(A R)^{2}$ is constant for the same material, ${ }^{19}$ the critical compression stress $S^{*}$ in the case of nanolines should be $\approx 30$ times higher than that of the nanowalls, in agreement with the absence of significant deformation in Figure $4 \mathrm{~d}$. Thus, nanolines can accommodate higher stress than nanowalls. To qualitatively capture the band structure shown in Figure 4g, faster sound velocities and hence higher Young modulus of nanolines are necessary compared to those for nanowalls (Table 2). This is in line with the expected increase of bending stiffness and the compressive residual stress of short $A R$ nanolines. ${ }^{11,36}$ The disparity of the mechanical strength $(\sim 15 \%)$ in the two photoresist gratings fabricated by the same original material clearly indicates the necessity of non-destructive metrology, in particular for nanostructures. And this is not restricted to a single physical property (Table 2).

The experimental dispersion of nanolines can still be captured by the guided Lamb modes of an infinite plate of the same width $w$ (Figure S1), in spite of their small $A R$. However, the suppression of extensional $\mathrm{S}_{0}$ mode in the nanoline spectrum is remarkable (Figure $4 \mathrm{f}$ ) in view of the strong intensity of $\mathrm{S}_{0}$ in high $A R$ nanowalls (Figure 3a). The justification of the $\mathrm{S}_{0}$ suppression involves a photoelastic coefficient ratio $P_{11} / P_{12}$ higher than in the nanowalls case ( 0.67 vs 0.10 in Table 2), despite being fabricated from the same original material. A possible reason for this elasto-optic modification could be related to the variation of the epoxy crosslinking characteristics, as a consequence of the sinusoidal interference light intensity, and the longer crosslinking times for short nanolines. ${ }^{12}$ As $P_{11}$ relates to displacements parallel to the polarization direction ( $y$-axis), i.e. flexural modes, a stronger crosslinked polymeric matrix could accommodate an increase of $P_{l l}$. While the photoelastic coefficients are well defined for crystalline materials, ${ }^{37}$ in the case of polymers subtle local structural changes can impart these coefficients. Again this disparity emphasizes the importance of material characterization at different length scales.

\section{Liquid filled gratings}

Based on the displacement field of the vibration modes in Figure 3d, the strain is localized inside the nanowall volume, while the substrate is essentially unstrained. In order to examine the phonon confinement along the buckled nanowalls, we recorded the dispersion relation with different orientations 
of $\mathbf{q}$ with respect to the wall direction, as illustrated in the inset to Figure 5. The superposition of the modes 1, 2 and 4 when their frequencies are plotted as a function of the projected $q_{\mathrm{x}}(=q \cdot \cos \varphi)$ evidences the guidance of the elastic excitations along the nanowalls. In fact, the computed density of states at several angles $\varphi$ (Figure S7) supports the experimental finding. This is an advantage when utilizing nanowalls as host for liquids; we emphasize that the main discrepancies between the empty and liquidfilled nanowalls are not due to the buckling, but entirely to the filling liquid.

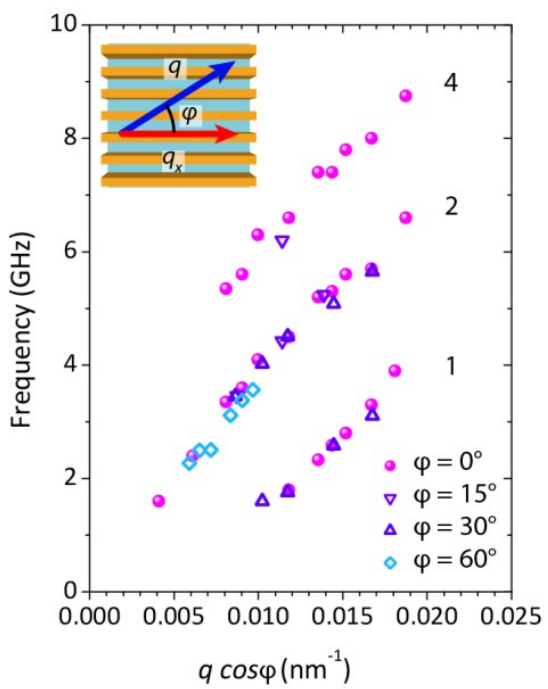

|Figure 5: Phonon guiding. Dispersion relation as a function of the projected $q_{x}$ along the nanowalls. The inset displays a top-view SEM micrograph, indicating that the direction of $q$ is tilted by an angle $\varphi$.

The phononic behavior of high AR gratings is expected to be significantly altered upon the presence of a different environment (i.e. liquid). We infiltrated the gratings with a refractive index matched liquid (Cargille Laboratories) that suppresses partially the diffracted beams in transmission geometry. In this case, it is possible to record the phonon propagation across the nanowalls ( $y$ direction). The infiltration of the grating with a liquid allows the propagation of the longitudinal acoustic wave along and across the nanowalls. Figure 6a displays the theoretical and experimental dispersion relation for propagation across the nanowalls (where the displacement has mainly $u_{\mathrm{y}}$ and $u_{\mathrm{z}}$ components) of Cargille-filled nanowalls. In this case, the grating behaves as $1 \mathrm{D}$ phononic crystal ${ }^{5}$ for longitudinal waves across the walls with the experimental points falling into the reduced $3 \mathrm{rd}$ and $4^{\text {th }}$ Brillouin zone. There is a deviation from the bulklike behavior (linear dispersion) caused by the periodicity in the sub micrometer range. The branches with the highest BLS intensities in Figure 6a relate to the well-known folded acoustic branches of an infinite 1D phononic crystal. Between these branches, additional branches with little contribution to BLS (dark blue dots in Figure 6a) correspond to stationary waves along the $z$-axis as a consequence of the finite size of the grating. The dispersion relation across the nanowalls ( $y$ axis) is qualitatively different from that along nanowalls ( $x$ axis, Figure 6 b) for which no band gap is predicted. However, the experimental 
dispersion could look similar (Figure S8) in view of the similar effective sound velocities in parallel ( $c_{\mathrm{eff}, \mathrm{x}}$ $=2130 \mathrm{~m} / \mathrm{s})$ and perpendicular $\left(c_{\text {eff,y }}=1880 \mathrm{~m} / \mathrm{s}\right)$ to the walls. In this case, the phonon guiding evidenced by Figure 5 is not apparent, and was confirmed by measurements (not shown here) at different orientation angles $\varphi$.

The dispersion relation along the nanowalls ( $x$-axis) is distinctly different in the two cases of empty (Figure 3c) and filled (Figure 6b) grating. While the former displays multiple branches corresponding to the propagation of phonons along the nanowalls, the dispersion relation of the latter has two branches of S-like character. The experiment captures only the upper one (Figure 6b), probably because the lower branch is obscured by the Rayleigh peak in the spectra. The higher orders contribute weakly to the BLS intensity. Note that in the FEM calculations of the infiltrated grating the effect of buckling was not simulated because first, the wavy patterns are random and second, the wavelength of the wavy patterns (d) is much longer in comparison with the phonon wavelength; these two factors cannot lead to an observable phononic effect. ${ }^{38}$ Hence, the simulations were performed with straight nanowalls. The elastic and photoelastic parameters needed for the theoretical modelling are the same in the filled and empty grating. The new introduced parameter is the photoelastic coefficient for the liquid $P=4$, which adopts a value higher than in the epoxy.
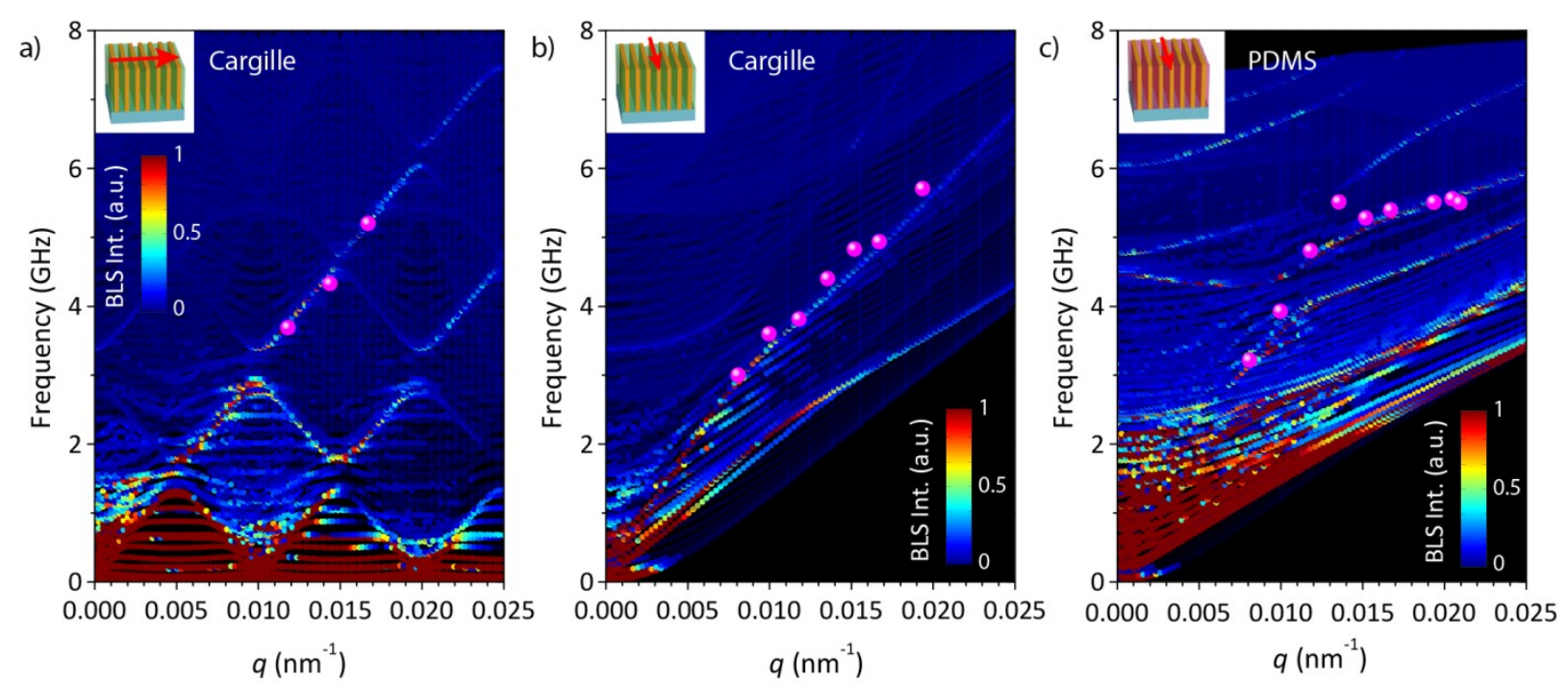

Figure 6: Infiltration. (a-b) Theoretical and experimental phononic dispersion relations (a) across and (b) along the high aspect ratio nanowalls infiltrated with Cargille liquid. (c) Theoretical and experimental phononic dispersion relations along PDMS-infiltrated nanowalls. PDMS is assumed to form a thin (10nm) solid ( $\mathrm{c}_{\mathrm{T}, \text { layer }}=250 \mathrm{~m} / \mathrm{s}$ ) layer around the nanowalls.

In order to verify the sensitivity of the grating to different infiltrating liquids, we filled a second sample of nanowalls with PDMS. In contrast to the previous case, the dispersion curves (Figure 6c) 
contain an almost flat branch around $5 \mathrm{GHz}$, observed also in other periodic structures infiltrated with PDMS. ${ }^{22}$ It is supposed that a very thin solidified layer of PDMS is formed under confinement. The flat branch in Figure 6c can be rationalized if we assumed that PDMS forms a solid layer in contact to the epoxy nanowalls of $10 \mathrm{~nm}$ thickness and transverse sound velocity $c_{\mathrm{T} \text {,layer }}=250 \mathrm{~m} / \mathrm{s}$. This theoretical flat branch in Figure 6c obtained in this way is associated with a strong displacement field in the solid PDMS layer. The susceptibility of the guided modes along nanowalls to the infiltrating liquid can be of unusual benefits: both sensing and alteration of the materials under confinement. ${ }^{39}$

\section{- CONCLUSIONS}

We have studied the elastic excitations of 1D epoxy-POSS gratings using BLS normal to the periodicity direction for two different ARs. Full FEM calculations yield the elastic and photoelastic parameters of the structure that are dependent on the fabrication conditions and aging. We demonstrated that the propagation of elastic waves along the epoxy nanowalls is preserved when the grating is in air despite buckling defects present in the high $A R$ nanowalls after aging. The liquid infiltration activates the propagation across the nanowalls. The recorded dispersion along the two main orthogonal directions $(x$ and $y$ ) is distinct, revealing anisotropy in the phonon propagation. The nanowall gratings are likely to be suitable not only as hosts for liquids but as nano-building blocks towards three-dimensional architected materials. ${ }^{40}$ Specifically, the assembly of building blocks at different length scales could lead to phonon filtering applications for stiffer structures. ${ }^{41-43}$

\section{- MATERIALS AND METHODS}

Fabrication of nano-gratings. The soft gratings were fabricated by two-beam interference lithography according to the procedure described in Ref. 10. In brief, the photoresist film was prepared from 50-70 wt $\%$ of epoxy polyhedral oligomeric silsesquoxane (epoxy-POSS, EP0408 from Hybrid Plastics) and 0.9 wt $\%$ (relative to the mass of epoxy-POSS) Irgacure 261 (visible photoacid generator, PAG, Ciba Specialty Chemicals). The exposed regions were crosslinked by baking at $50^{\circ} \mathrm{C}$ for $35 \mathrm{~s}$, followed by development in propylene glycol monomethyl ether acetate (PGMEA, Sigma-Aldrich) for $30 \mathrm{~min} .{ }^{12}$ To prevent the pattern collapse of the high aspect ratio nanolines, we rinsed the sample in isopropanol (IPA, SigmaAldrich) for $30 \mathrm{~min}$, followed by drying in $\mathrm{CO}_{2}$ supercritical point dryer (SAMDRI ${ }^{\circledR}-\mathrm{PVT}-3 \mathrm{D}$, Tousimis). The incident angles of the beams determine the periodicity and the height of the nanolines (i.e. film thickness) could be fine-tuned by spin coating speed. Here, we investigated two of them, $A R=h / w=2$ (nanolines) and 11 (nanowalls). 
Scanning electron microscopy. The morphology and dimensions of the nanowalls and nanolines were characterized by a scanning electron microscope (SEM, LEO Gemini 1530) at $0.7 \mathrm{kV}$.

Brillouin light scattering. BLS is a non-invasive technique which utilizes the scattering of an incident probing laser beam from thermally activated density fluctuations (phonons) in transparent materials along a certain direction. In transmission geometry, the probing wave vector orientation respect to the nanowalls $\alpha$ is selected with the scattering geometry, and its wavenumber $q=4 \pi / \lambda \sin (\theta / 2)$ is dependent on the wavelength of the probing beam $\lambda=532 \mathrm{~nm}$ and the scattering angle $\theta$ for propagation parallel to the substrate plane. The BLS spectrum consists of a family of frequency doublets at GHz frequencies, resolved by a tandem Fabry-Perót interferometer (JRS Instruments). In backscattering (BS) geometry, the wavenumber $q=4 \pi / \lambda_{\mathrm{BS}} \sin (\alpha)\left(\lambda_{\mathrm{BS}}=514.5 \mathrm{~nm}\right)$ depends on the angle of incidence $\alpha$ respect to the normal of the film.

Theoretical modeling. The dispersion curves $f(\mathbf{q})$ of the nanowalls are obtained by solving the elasticity equations of motion by using the finite element method (FEM). Due to the periodicity of the structure along $y$ direction, the problem is solved in one unit cell by assuming periodic boundary conditions at the limits of the unit cell. Free stress boundary conditions are used at the surfaces of the ridge which are in contact with vacuum whereas fixed boundary conditions are used at the bottom surface of the glass substrate (assumed to be of finite thickness). In the liquid-filled gratings, the boundary conditions at the liquid/solid interface are continuous for the normal stress and the normal displacement, given by

$\nabla p=\rho(2 \pi f)^{2} u$. From the knowledge of the eigenvalues and eigenvectors of the structure, one can calculate the BLS spectra based on Eqs. 2 and 3. The calculations were performed using the parameters in Table 2; otherwise is stated.

\section{- ACKNOWLEDGMENTS}

A.G and B.D.R thank Dr. E.H. El Boudouti for several helpful discussions about the theoretical calculations of the BLS spectra. EAR and GF acknowledge the support from ERC SuPro 340391 and ERC SmartPhon 694977, respectively. BG and CMST gratefully acknowledge support from the EC project MERGING (GA nr. 309150), the Spanish project PHENTOM (FIS 2015-70862P) and the Severo Ochoa Program (MINECO, Grant SEV-2013-0295). BG acknowledges Alexander von Humboldt foundation for support. 


\section{- REFERENCES}

1. Wang, Z. B.; Helander, M. G.; Qiu, J.; Puzzo, D. P.; Greiner, M. T.; Hudson, Z. M.; Wang, S.; Liu, Z. W.; Lu, Z. H., Nat. Photonics 2011, 5 (12), 753-757.

2. Kang, H.; Jung, S.; Jeong, S.; Kim, G.; Lee, K., Nat. Comm. 2015, 6, 6503.

3. Papadopoulos, P.; Deng, X.; Mammen, L.; Drotlef, D. M.; Battagliarin, G.; Li, C.; Mullen, K.; Landfester, K.; del Campo, A.; Butt, H. J.; Vollmer, D., Langmuir 2012, 28 (22), 8392-8398.

4. Neuhaus, S.; Spencer, N. D.; Padeste, C., ACS Appl. Mater. Interfaces 2012, 4 (1), 123-130.

5. Schneider, D.; Liaqat, F.; El Boudouti, E. H.; El Hassouani, Y.; Djafari-Rouhani, B.; Tremel, W.; Butt, H. J.; Fytas, G., Nano Lett. 2012, 12 (6), 3101-3108.

6. David, C.; Bruder, J.; Rohbeck, T.; Grünzweig, C.; Kottler, C.; Diaz, A.; Bunk, O.; Pfeiffer, F., Microelectron. Eng. 2007, 84 (5-8), 1172-1177.

7. Graczykowski, B.; Sledzinska, M.; Kehagias, N.; Alzina, F.; Reparaz, J. S.; Sotomayor Torres, C. M. Appl. Phys. Lett. 2014, 104, 123108.

8. Choi, Y.; Jeon, H.; Kim, S. Lab Chip 2015, 15, 642.

9. Tseng, Y.-C.; Mane, A. U.; Elam, J. W.; Darling, S. B., Adv. Mater. 2012, 24, 2608-2613.

10. Zhang, Y.; Lo, C.-W.; Taylor, J. A.; Yang, S., Langmuir 2006, 22, 8595-8601.

11. Kalima, V.; Siitonen, S.; Karvinen, P.; Suvanto, M.; Kuittinen, M.; Pakkanen, T. T., J Micromech. Microeng. 2008, $18(2), 025020$.

12. Li, J.; Yang, S., Fabrication of High-Aspect-Ratio (up to 10). Microelectron. Eng. 2014, 128, 7-11.

13. Li, J.; Cho, Y.; Choi, I.-S.; Yang, S., Adv. Funct. Mater. 2014, 24 (16), 2361-2366.

14. Delcambre, S. P.; Riggleman, R. A.; de Pablo, J. J.; Nealey, P. F., Soft Matter 2010, 6 (11), 2475.

15. Liu, Y.; Chen, Y.-C.; Hutchens, S.; Lawrence, J.; Emrick, T.; Crosby, A. J., Macromolecules 2015, 48 (18), 6534-6540.

16. O'Connell, P. A.; McKenna, G. B., Science 2005, 307, 1760-1763.

17. Ayouch, A.; Dieudonné, X.; Vaudel, G.; Piombini, H.; Vallé, K.; Gusev, V.; Belleville, P.; Ruello, P., E, ACS Nano 2012, 6 (12), 10614-10621.

18. Poyser, C. L.; Czerniuk, T.; Akimov, A.; Diroll, B. T.; Gaulding, E. A.; Salasyuk, A. S.; Kent, A. J.; Yakovlev, D. R.; Bayer, M.; Murray, C. B., ACS Nano 2016, 10 (1), 1163-1169. 
19. Tirumala, V. R.; Stafford, C. M.; Ocola, L. E.; Douglas, J. F.; Mahadevan, L., Nano Lett. 2012, 12 (3), 15161521.

20. Schneider, D.; Gomopoulos, N.; Koh, C. y.; Papadopoulos, P.; Kremer, F.; Thomas, E. L.; Fytas, G., Nat. Mater. 2016, in press.

21. Paven, M.; Fuchs, R.; Yakabe, T.; Vollmer, D.; Kappl, M.; Itakura, A. N.; Butt, H.-J., Adv. Funct. Mater. 2016, 26

4914-4922.

22. Sato, A.; Pennec, Y.; Shingne, N.; Thurn-Albrecht, T.; Knoll, W.; Steinhart, M.; Djafari-Rouhani, B.; Fytas, G., ACS Nano 2010, 4 (6), 3471-3481.

23. Cheng, W.; Sainidou, R.; Burgardt, P.; Stefanou, N.; Kiyanova, A.; Efremov, M.; Fytas, G.; Nealey, P. F., Macromolecules 2007, 40 (20), 7283-7290.

24. Regonda, S.; Aryal, M.; Hu, W., J. Vac. Sci. Technol., B: Microelectron. Nanometer Struct.--Process., Meas., Phenom. 2008, 26 (6), 2247-2251.

25. Yang, S.; Megens, M.; Aizenberg, J.; Wiltzius, P.; Chaikin, P. M.; Russel, W. B., Chem. Mater. 2002, 14 (7), 2831-2833.

26. Hartschuh, R. D.; Kisliuk, A.; Novikov, V.; Sokolov, A. P.; Heyliger, P. R.; Flannery, C. M.; Johnson, W. L.; Soles, C. L.; Wu, W. L. Appl. Phys. Lett. 2005, 87, 173121.

27. Bandhu, R. S.; Sooryakumar, R.; Bussmann, K., Ann. Phys. 2011, 523 (1-2), 107-120.

28. Jiménez Riobóo, R. J.; Sánchez-Sánchez, A.; Prieto, C. Phys. Rev. B 2016, 94, 014313.

29. Arechhi, F. T.; Schulz-Dubois, E. O., Laser Handbook; North-Holland, 1972.

30. Royer, D.; Dieulesaint, E., Elastic Waves in Solids; Springer, 2010.

31. Rakhymzhanov, A. M.; Gueddida, A.; Alonso-Redondo, E.; Utegulov, Z. N.; Perevoznik, D.; Kurselis, K.; Chichkov, B. N.; Boudouti, E. H. E.; Djafari-Rouhani, B.; Fytas, G., Appl. Phys. Lett. 2016, 108, 201901.

32. Stan, G.; Ciobanu, C. V.; Levin, I.; Yoo, H. J.; Myers, A.; Singh, K.; Jezewski, C.; Miner, B.; King, S. W., Nano Lett. 2015, 15 (6), 3845-3850.

33. Alvine, K. J.; Ding, Y.; Douglas, J. F.; Ro, H. W.; Okerberg, B. C.; Karim, A.; Soles, C. L., Soft Matter 2009, 5 (15), 2913.

34. Ishiyama, C.; Higo, Y., J. Polym. Sci., Part B: Polym. Phys. 2002, 40 (5), 460-465.

35. Abedinnasab, M. H.; Hussein, M. I., Wave Motion 2013, 50 (3), 374-388. 
36. Tan, J. L.; Tien, J.; Pirone, D. M.; Gray, D. S.; Bhadriraju, K.; Chen, C. S., Proc. Natl. Acad. Sci. U SA 2003, $100(4), 1484-1489$.

37. Higginbotham, C. W.; Cardona, M.; Pollak, F. H., Phys. Rev. 1969, 184 (3), 821-829.

38. Rudykh, S.; Boyce, M. C., Phys. Rev. Lett. 2014, 112 (3), 034301.

39. Amoudache, S.; Pennec, Y.; Rouhani, B. D.; Khater, A.; Lucklum, R.; Tigrine, R., J. Appl. Phys. 2014, 115 (13), 134503.

40. Montemayor, L.; Chernow, V.; Greer, J. R., MRS Bull. 2015, 40 (12), 1122-1129.

41. Davies, B.; King, A.; Newman, P.; Minett, A.; Dunstan, C. R.; Zreiqat, H., Sci. Rep. 2014, 4, 7538.

42. Xu, Y. L.; Tian, X. G.; Chen, C. Q., Phys. B 2012, 407 (12), 1995-2001.

43. Guerder, P.-Y.; Deymier-Black, A. C.; Swinteck, N. Z.; Vasseur, J. O.; Bou-Matar, O.; Muralidharan, K.; Deymier, P. A., J. Mech. Behav. Biomed. Mater. 2014, 37, 24-32. 


\section{Directional Elastic Wave Propagation in High-Aspect-Ratio Photoresist Gratings: Liquid Infiltration and Aging}

E. Alonso-Redondo, ${ }^{1}$ A. Gueddida, ${ }^{2,3}$ J. Li, ${ }^{4}$ B. Graczykowski, ${ }^{5}$ C. M. Sotomayor Torres,${ }^{5,6}$ Y.Pennec, ${ }^{2}$ S. Yang, ${ }^{4}$ B. Djafari-Rouhani, ${ }^{2}$ and G. Fytas ${ }^{1,7, *}$

\section{TABLE OF CONTENTS}

The directional elastic wave propagation in high-aspect-ratio photoresist gratings is assessed by Brillouin spectroscopy in two cases: liquid infiltration and aging.
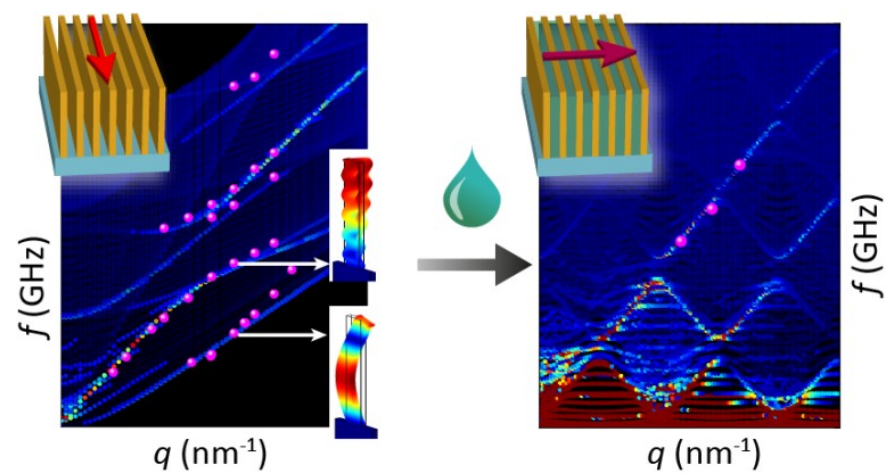


\section{Directional Elastic Wave Propagation in High-Aspect-Ratio Photoresist Gratings: Liquid Infiltration and Aging}

E. Alonso-Redondo, A. Gueddida, J. Li, B. Graczykowski, C. M. Sotomayor Torres, Y.Pennec, S. Yang, B. Djafari-Rouhani, and G. Fytas

\section{SUPPLEMENTARY INFORMATION}

\section{Periodicity modes}

An in situ characterization of the periodicity of the grating was performed through the scattering of the diffracted beams in transmission geometry. The strongly diffracted beams act as input beams for subsequent scattering in the glass substrate by the bulk longitudinal and transverse phonons. Their frequency shift is described by

$$
f(k)=\frac{C_{\text {glass }}}{2 \pi} \sqrt{G^{2}+k^{2} \pm 2 \varphi r k s i n} .
$$

Here $G=2 \pi / a$ is the reciprocal lattice constant, $\varphi$ is the relative angle between the probing wave vector $\mathbf{q}$ and the nanowalls (Figure 5 inset) and $c_{\text {glass }}$ is the sound velocity in the glass substrate. Equation 1 is obtained from $f=c_{\text {glass }} q / 2 \pi$, with $q^{2}=q_{\mathrm{x}}{ }^{2}+q_{\mathrm{y}}{ }^{2}$, and $q_{\mathrm{y}}=k \pm G$, due to the momentum conservation. ${ }^{1}$ For propagation along the nanowalls $(\varphi=0)$, there are two branches $\left(p_{\mathrm{L}}\right.$ and $p_{\mathrm{T}}$ in Figure $\left.2 \mathrm{c}\right)$ dependent on substrate sound velocity, either the longitudinal or the transverse (Table 2). If $q$ has a $y$-component $(\varphi \neq 0)$ with an orientation as small as $1^{\circ}$, each "periodicity peak" in the BLS spectrum splits into a doublet; thus there are four branches in the dispersion plot. The sensitivity of the spectrum to the nanowall orientation in the scattering plane allows a precise control on the $q$-direction. Representation of the experimental points by Supplementary Eq. 1 (solid lines in Figure 2c) using fixed the known values of the two glass sound velocities yields the lattice parameter $a$.

\section{Band sorting method}

The calculation of the dispersion curves from which the densities of states and Brillouin scattering spectra can be deduced based on the finite element method (FEM, see Methods). Since the material of nanowalls have very different acoustic impedance $(Z=c \rho)$ than the substrate, we shall be mostly interested in the modes below the sound lines of the glass substrate (red lines in Figure S1a) which are highly confined inside the nanowalls and cannot penetrate into the substrate. Still, due to the finite size of the nanowalls and nanolines, there are a great number of dispersion curves below the glass sound lines. One can make a selection of the modes for which the displacement field is mainly composed of $u_{x}$ and $u_{y}$ components. This selection is based on a band sorting method by means of the following relation 


$$
P_{x y}=\frac{\iiint_{\text {Nanowalls }}\left(\left|u_{x}\right|^{2}+\left|u_{y}\right|^{2}\right) d v}{\iiint_{\text {unitcell }}\left(\left|u_{x}\right|^{2}+\left|u_{y}\right|^{2}+\left|u_{z}\right|^{2}\right) d v}
$$

This relation means that the mode which have mainly $u_{x}$ and $u_{y}$ components display a $P_{x y}$ close to 1 , whereas the modes with $u_{z}$ component have $P_{x y}$ close to 0 . Figure S1b shows the dispersion branches that satisfy to the condition $P_{x y} \geq 0.8$. One can recognize a similarity between these modes and those of an infinite plate of equal thickness as the nanowall (Figure S2a). Therefore we call them $S_{n}$ and $A_{n}$ like ( $n$ is an integer) for the modes whose displacement fields are respectively symmetric and antisymmetric with respect to the mid-plane of the plate.

\section{Morphological effects}

To examine whether morphological changes of the nanolines and nanowalls relate to the observed change of the elastic parameters, we consider the effect of the grating wall shape. Deviation from the theoretical rectangular shape is feasible in the soft matter. We consider two deviations from the ideal straight rectangular shape: (i) round top and round grafting of the nanolines to the substrate (Figure S4) and (ii) trapezoidal nanowalls being narrower on the top than at the bottom (Figure S5). The BLS intensities and the dispersion relation are not significantly affected by including a small rounding either on the top (Figure S4b) or at the bottom (Figure S4c) of the nanowall structure. ${ }^{2}$ The second shape defect (trapezoidal) can relate to the sinusoidal profile of the interference light pattern. Only above a critical light intensity the oligomers will be crosslinked; hence the bottom of the nanowalls should have larger crosslinking area than the top, leading to a broader bottom of the nanowalls. The computed BLS intensitydispersion relation of a trapezoidal nanowall can be understood as the superposition of the dispersions by a nanowall with thickness in a range $100-250 \mathrm{~nm}$. The $\mathrm{S}_{0}$ and $\mathrm{S}_{1}$-like bands are broad because the phasematching frequency sweeps the acoustic dispersion curve as the width of the nanowall increases. That creates an apparent blue-shift of the S-like modes (Figure S5a). The increase of the sound velocities, in general, causes a blue-shift of the modes (Figure S6) but it does not suffice to completely capture the blue shift of the resolved acoustic branches. More specifically, the $\mathrm{S}_{0}$-like mode is sensitive to $c_{\mathrm{T}}$ as well as the $\mathrm{S}_{1}$-like mode but at higher wave vectors (Figure S6). In contrast, the increase of $c_{\mathrm{L}}$ mostly produces a blue shift of only $\mathrm{S}_{1}$ and $\mathrm{S}_{2}$-like modes. The trapezoidal shape, together with the slight increase in velocities is necessary to completely describe the experimental dispersion. In conclusion, the phononic dispersion of nanowalls is very sensitive to variations in the wall thickness, but not variations of their edges shape. 

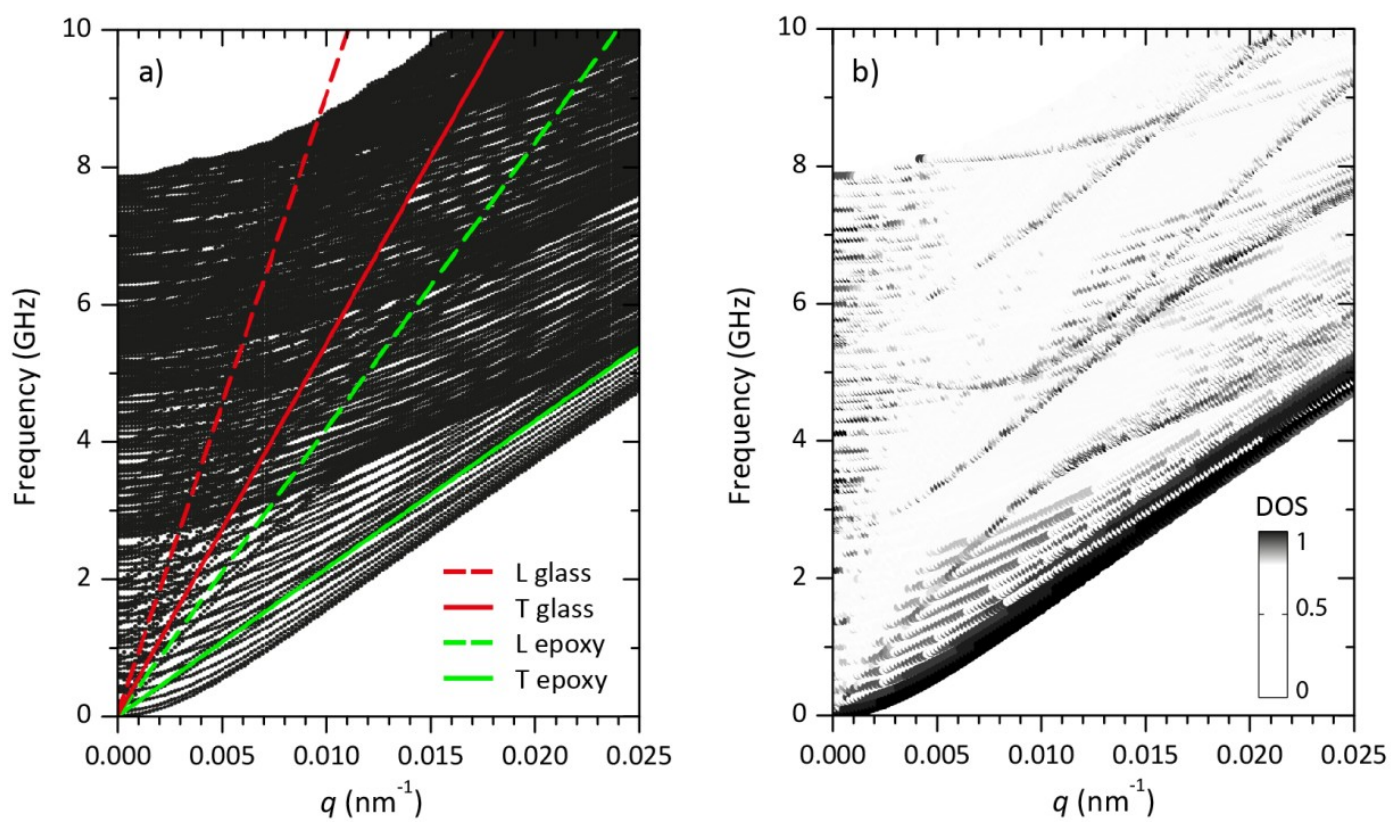

Figure S1: (a) Full dispersion curves of nanowalls calculated for their 300 first eigenfrequencies. (b) Dispersion curves of the modes with mainly $u_{\mathrm{x}}$ and $u_{\mathrm{y}}$ components $\left(P_{x y} \geq 0.8\right)$.
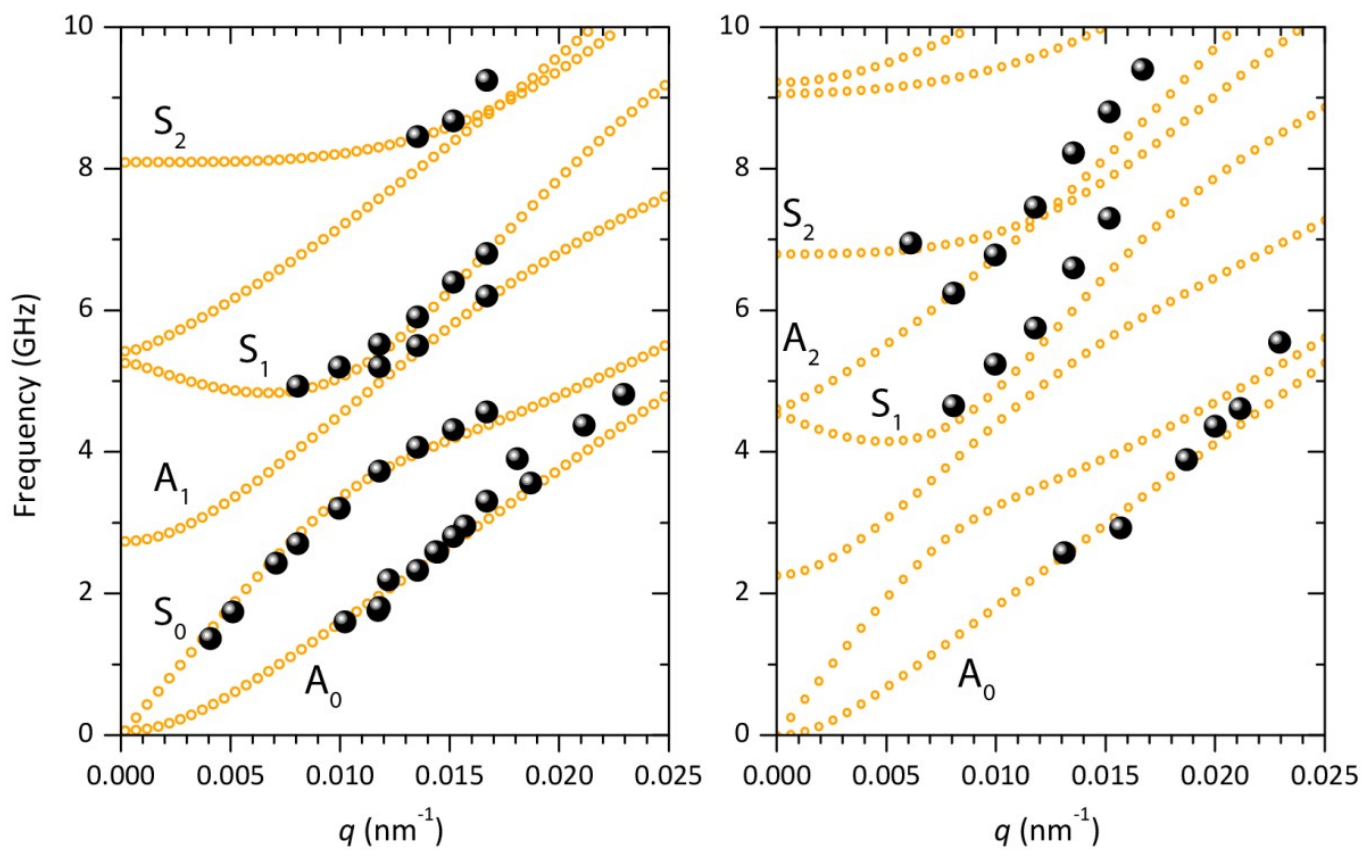

Figure S2: Lamb plate theoretical dispersion plot (orange open circles) and experimental dispersion (black spheres) for (a) high aspect ratio nanowalls and (b) short aspect ratio nanolines. The experimental points have been acquired in both T and BS geometry. 

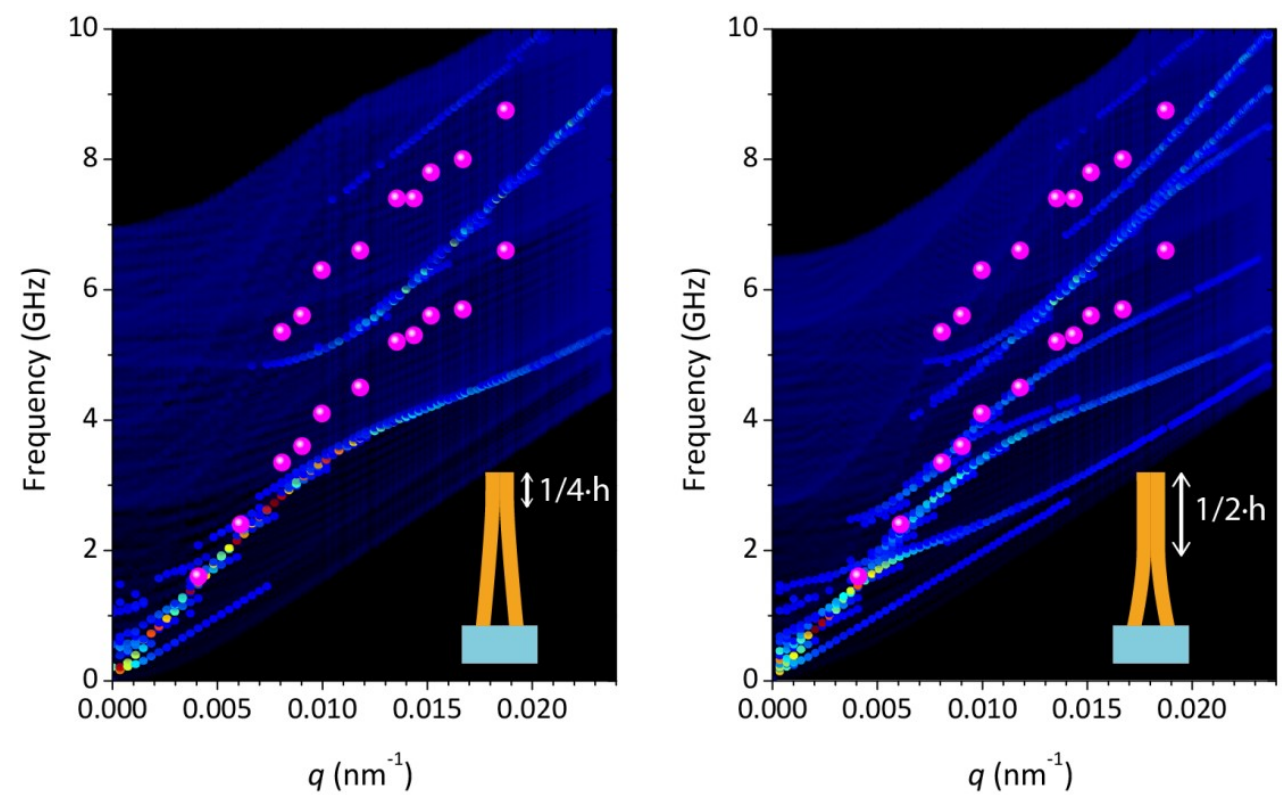

Figure S3: Effect of the contact between high aspect ratio nanowalls in the VV BLS intensity: (a) small contact area, nanowalls touch about $1 / 4$ of their height; (b) large contact area, nanowalls touch about $1 / 2$ of their height. The bands of greater intensity in (b) correspond to the Lamb modes of a plate of $2 w$ width.

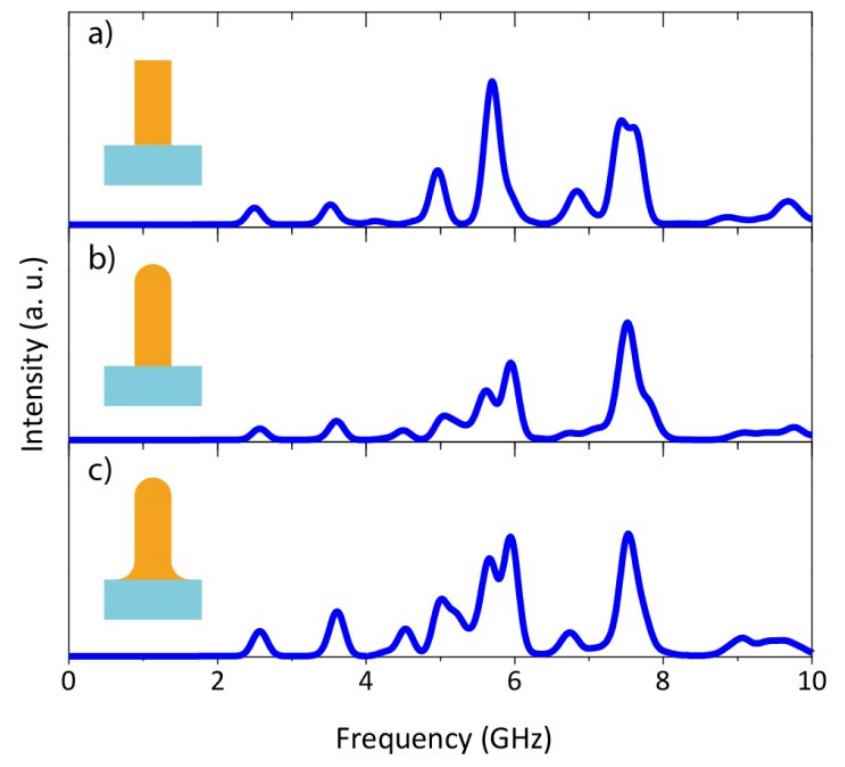

Figure S4: Theoretical BLS spectra at $q=0.0118 \mathrm{~nm}^{-1}$ as a function of the nanoline section shape. 

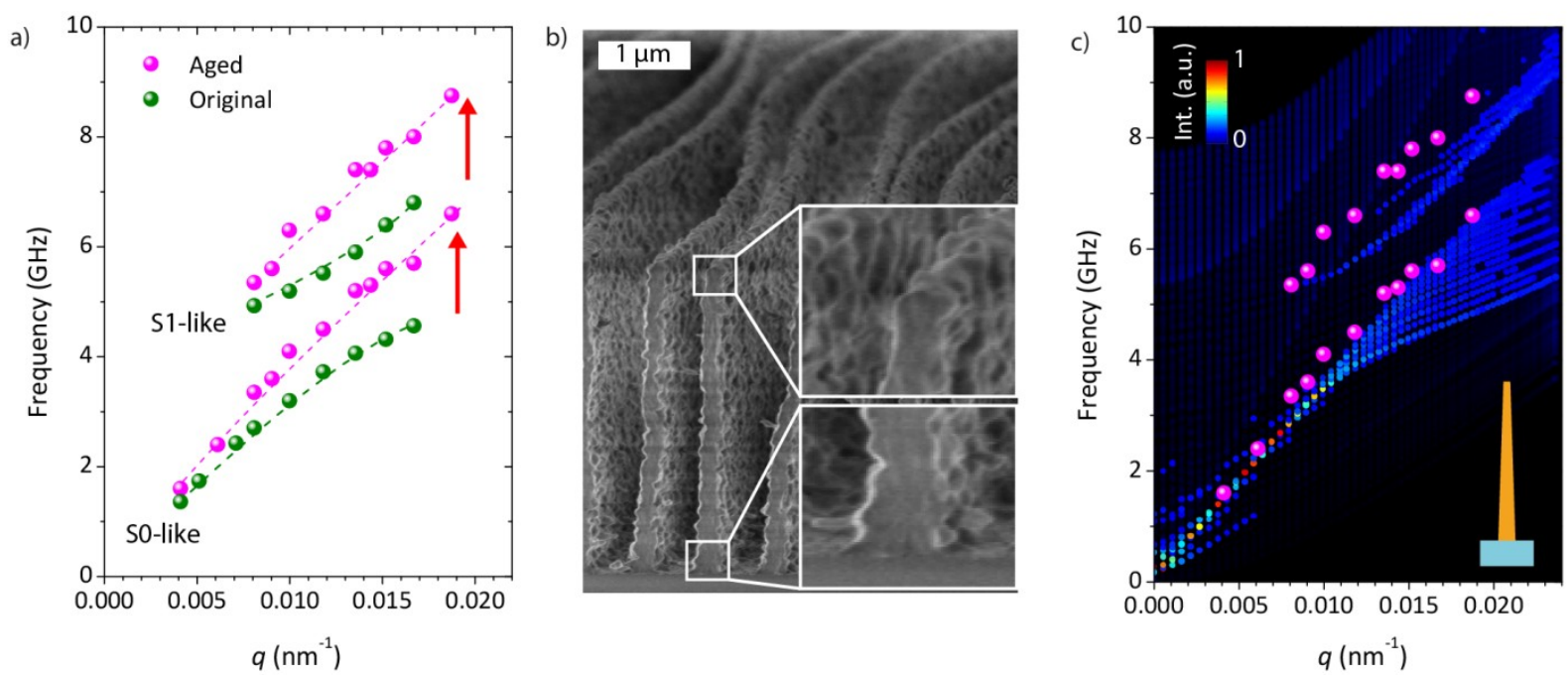

Figure S5: (a) Experimental dispersion plot (VV polarization) of trapezoidal aged nanowalls (pink) is blue-shifted respect to the original (green). (b) Cross section SEM micrograph displays the distinct width at the base (bottom inset) and the top (upper inset) of the nanowalls. (c) Dispersion relation colored according to the VV BLS intensity.
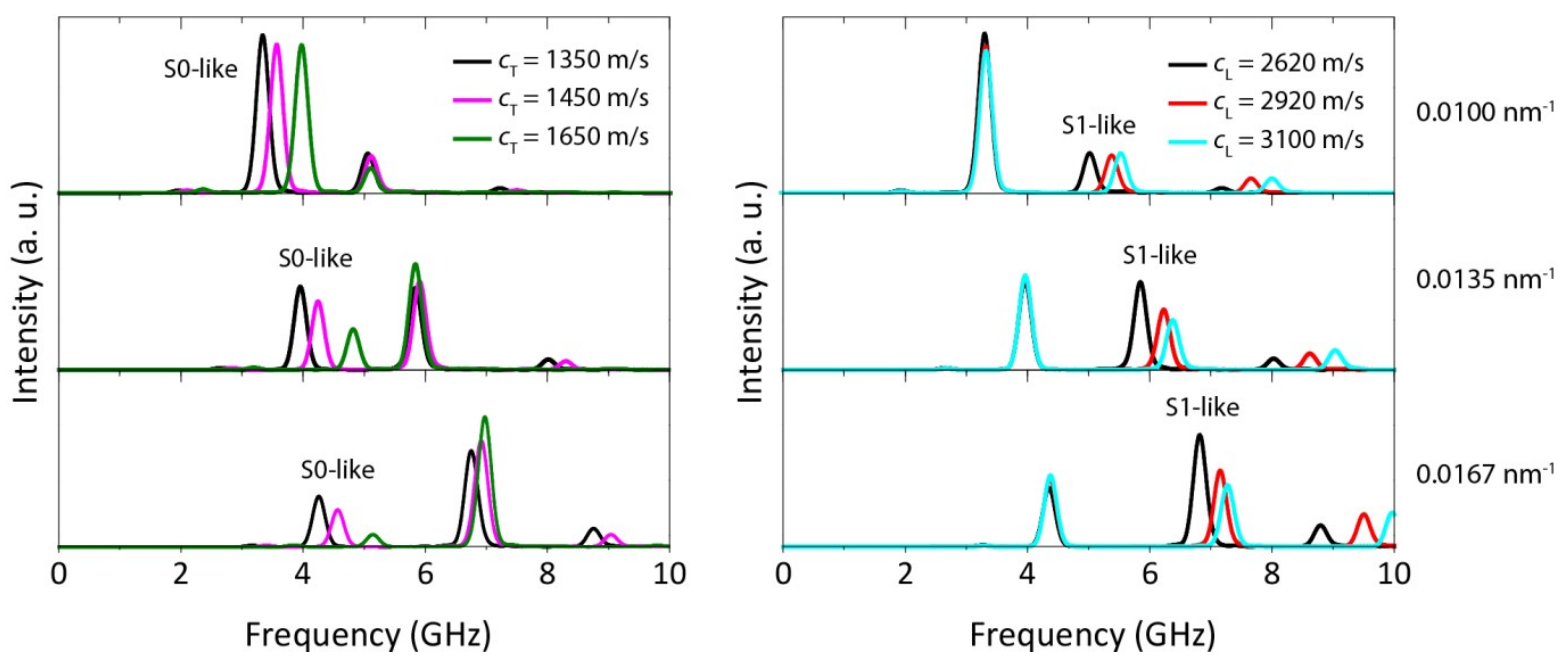

Figure S6: (a-b) Effect of (a) $c_{\mathrm{T}}$ and (b) $c_{\mathrm{L}}$ in high $A R$ nanowalls BLS intensity. S0-like mode is sensitive mainly to $c_{\mathrm{T}}$ a while S1-like mode is sensitive to $c_{\mathrm{L}}$ and to $c_{\mathrm{T}}$ in lesser degree. The sound velocity has been fixed to $c_{\mathrm{L}}=2620$ $\mathrm{m} / \mathrm{s}$ in (a) and $c_{\mathrm{T}}=1350 \mathrm{~m} / \mathrm{s}$ in (b). 

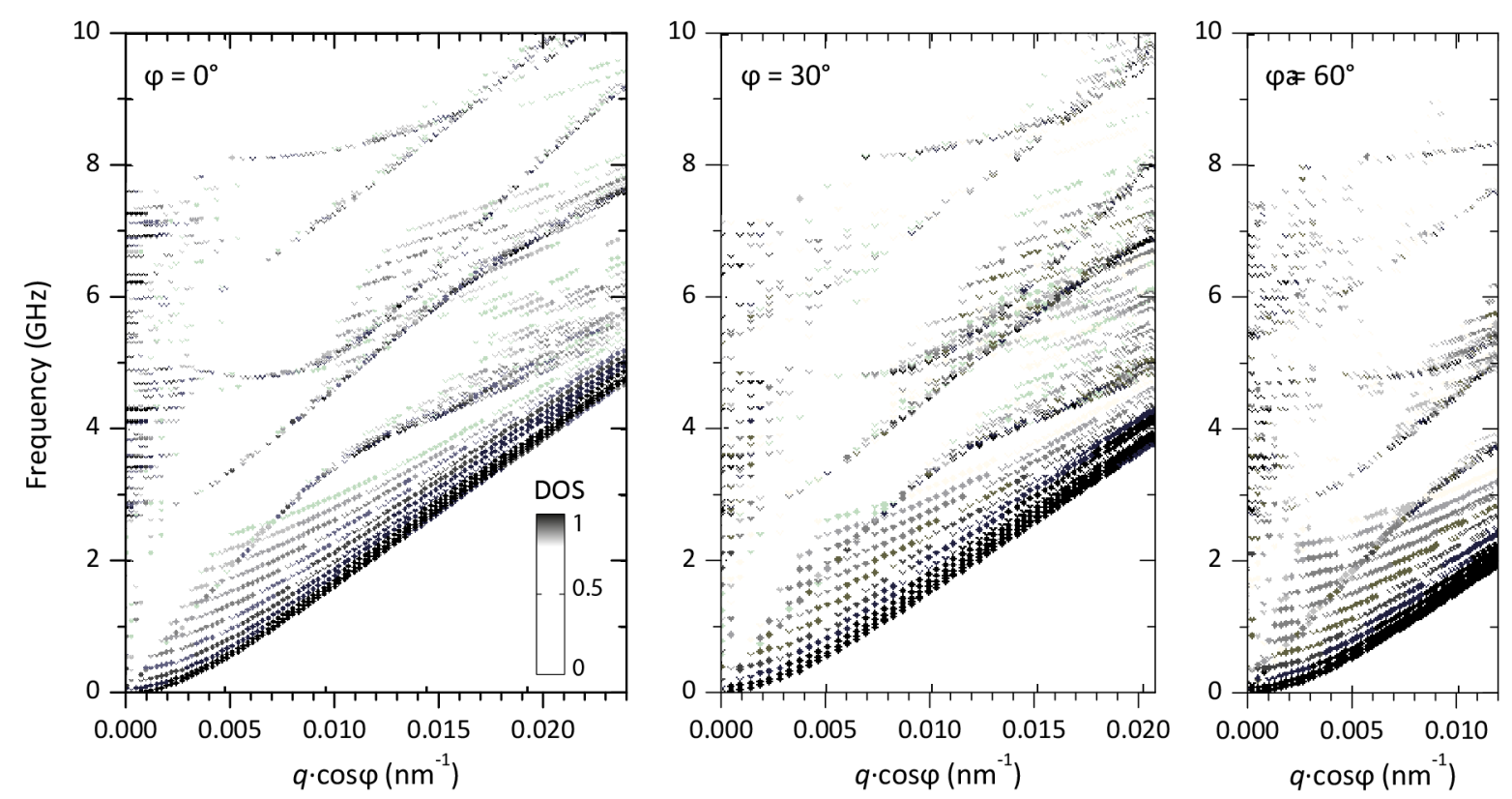

Figure S7: Normalized density of states (DOS) for long nanowalls, as a function of the projected $q$ along the nanowalls, for three different orientations $\varphi$.
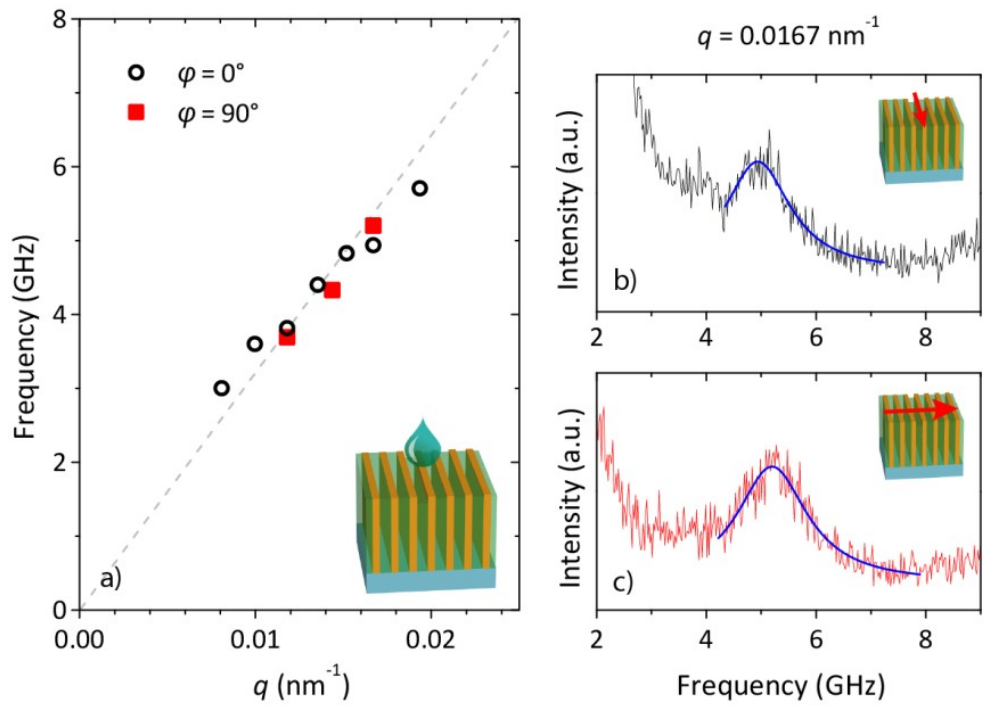

Figure S8: (a) Experimental dispersion relation measured with probing $q$ along $\left(\varphi=0^{\circ}\right)$ and perpendicular $\left(\varphi=90^{\circ}\right)$ to the nanowalls of the grating filled with Cargille liquid. The dashed line is a guide for the eye. (b-c) VV spectra at $0.0167 \mathrm{~nm}^{-1}$ (b) along and (c) across the nanowalls. 


\section{- SUPPLEMENTARY REFERENCES}

1. Urbas, A. M.; Thomas, E. L.; Kriegs, H.; Fytas, G.; Penciu, R. S.; Economou, L. N., Phys. Rev. Lett. 2003, 90 (10), 108302.

2. Johnson, W. L.; Kim, S. A.; Geiss, R.; Flannery, C. M.; Soles, C. L.; Wang, C.; Stafford, C. M.; Wu, W. L.; Torres, J. M.; Vogt, B. D.; Heyliger, P. R., Nanotechnology 2010, 21 (7), 75703. 